PHYSICAL REVIEW D 81, 091502(R) (2010)

\title{
Quarkyonic phase in dense two color matter
}

\author{
Simon Hands \\ Department of Physics, Swansea University, Singleton Park, Swansea SA2 8PP, United Kingdom \\ Seyong Kim \\ Department of Physics, Sejong University, Seoul 143-747, Korea \\ Jon-Ivar Skullerud \\ Department of Mathematical Physics, National University of Ireland Maynooth, Maynooth, County Kildare, Ireland
}

(Received 11 January 2010; published 20 May 2010)

\begin{abstract}
We present results from simulations of two color QCD with two Wilson quark flavors in the presence of a quark chemical potential $\mu$ at two different lattice spacings. The equation of state, conformal anomaly, superfluid order parameter, and Polyakov line are all discussed. Our results suggest that the transitions from hadronic to quark matter and from confined to deconfined matter occur at distinct values of $\mu$, consistent with the existence of a quarkyonic phase in this model.
\end{abstract}

DOI: 10.1103/PhysRevD.81.091502

PACS numbers: 11.15.Ha, 12.38.Aw, 21.65.Qr

A quantitative understanding of cold dense baryonic matter, i.e. the region of the $(T, \mu)$ plane conventionally placed at the lower right of the QCD phase diagram (here $\mu$ is the quark chemical potential), remains an outstanding problem in theoretical physics. There has been much discussion of possible exotic color superconducting (CSC) phases in this region, where color-carrying degrees of freedom such as quarks or gluons are all gapped via a Higgs-Meissner mechanism, and which may also be superfluid or even crystalline [1]. This picture has its firmest theoretical support at weak gauge coupling, where superconductivity is understood as being due to the BCS condensation of diquark Cooper pairs. More recently, an alternative scenario based on large- $N_{c}$ arguments has emerged, whose ground state at sufficiently high density has a restored chiral symmetry, so that thermodynamic properties are well described by a degenerate relativistic Fermi sea of quarks characterized by a Fermi momentum $k_{F}$, but which remains in a confined "quarkyonic" phase so that all excitations necessarily carry color-singlet quantum numbers [2].

It remains unclear whether the CSC and quarkyonic pictures are truly distinct or are in some sense complementary. However, it is salutary to recall that even basic questions involving dense matter, such as the maximum stable mass of a neutron star, need quantitative input about the equation of state of ultradense matter regardless of the nature of the ground state; this requires a controlled nonperturbative calculation.

The most reliable source of such information, lattice $\mathrm{QCD}$, is in general inoperable in this regime for the following reason. In Euclidean metric the QCD Lagrangian density for quarks reads

$$
\mathcal{L}_{\mathrm{QCD}}=\bar{\psi}\left(\not D[A]+\mu \gamma_{0}+m\right) \psi \equiv \bar{\psi} M \psi .
$$

It is straightforward to show $\gamma_{5} M(\mu) \gamma_{5} \equiv M^{\dagger}(-\mu)$, im- plying $\operatorname{det} M(\mu)=(\operatorname{det} M(-\mu))^{*}$, and therefore that the path integral measure is not real and positive for $\mu \neq 0$. Monte Carlo importance sampling, the mainstay of numerical lattice QCD, is ineffective. It is helpful to consider what goes wrong if the real positive measure factor $\operatorname{det} M^{\dagger} M$ as implemented in, e.g. the hybrid Monte Carlo (HMC) algorithm, is used. In QCD, while $M$ describes a color triplet of quark fields $q$, the $M^{\dagger}$ factor describes color antitriplet "conjugate quarks" $q^{c}$. Gauge singlet bound states of the form $q q^{c}$ resemble mesons, but carry nonzero baryon charge $B>0$. The lightest such state is degenerate with the pseudo-Goldstone $\pi$ meson; hence HMC simulations with $\mu \neq 0$ predict an unphysical "onset" transition from the vacuum to a state with quark density $n_{q}>0$ at $\mu_{o} \simeq \frac{1}{2} m_{\pi}$. The resulting ground state is a Bose-Einstein condensate (BEC) of diquark baryons, bearing no resemblance to nuclear matter, which phenomenologically we know forms at $\mu_{o} \approx \frac{1}{N_{c}} m_{\text {nucleon. The physical transition }}$ can be found only if the correct complex path integral measure $\operatorname{det}^{2} M$ is used, and it must result from extremely nontrivial cancellations between configurations with differing phases-this has come to be known as the Silver Blaze problem [3].

In this paper we consider an alternative strongly interacting theory, two color $\mathrm{QCD}\left(\mathrm{QC}_{2} \mathrm{D}\right)$, in which the gauge group is $\mathrm{SU}(2)$. Since $q$ and $\bar{q}$ live in equivalent representations of $\mathrm{SU}(2)$, it follows that $\operatorname{det} M(\mu) \equiv \operatorname{det} \tau_{2} M^{*}(\mu) \tau_{2}$ is real, and therefore the theory has a positive measure for an even number $N_{f}$ of quark flavors [4]. Physically this is expressed through both $q \bar{q}$ mesons and $q q, \bar{q} \bar{q}$ baryons falling in the same hadron multiplets. For sufficiently light quarks the scale hierarchy $m_{\pi} \ll m_{\rho}$ permits the use of chiral perturbation theory $(\chi \mathrm{PT})$ in studying the response of the lightest multiplet to $\mu \neq 0$ [5]. The key result is that for $\mu \geq \mu_{o} \equiv \frac{1}{2} m_{\pi}$ a nonzero baryon charge density $n_{q}>$ 0 does develop, along with a gauge-invariant superfluid 
order parameter which for $N_{f}=2$ reads $\langle q q\rangle \sim$ $\left\langle\psi^{\mathrm{tr}} C \gamma_{5} \tau_{2} \epsilon_{a b} \psi\right\rangle \neq 0$, where $\tau_{2}$ acts on color indices and $\epsilon_{a b}=-\epsilon_{b a}$ on flavor. The resulting BEC is composed of weakly interacting $q q$ baryons with $J^{P}=0^{+}$.

For $\mu \geq \mu_{o}$ leading-order $\chi \mathrm{PT}$ predicts a smooth rotation of the chiral condensate $\langle\bar{\psi} \psi\rangle$ into the superfluid condensate $\langle q q\rangle$ as $\mu$ increases [5]. In addition, there is a quantitative prediction for quark density:

$$
n_{q}(\mu)=8 N_{f} f_{\pi}^{2} \mu\left(1-\frac{\mu_{o}^{4}}{\mu^{4}}\right)
$$

where the parameters $\mu_{o}$ and $f_{\pi}$ suffice to specify $\chi \mathrm{PT}$ at this order. It is possible to develop the thermodynamics of the system at $T=0$ more fully, to extract pressure and energy density [6]:

$$
\begin{gathered}
p_{\chi \mathrm{PT}}=\int_{\mu_{o}}^{\mu} n_{q} d \mu=4 N_{f} f_{\pi}^{2}\left(\mu^{2}+\frac{\mu_{o}^{4}}{\mu^{2}}-2 \mu_{o}^{2}\right) ; \\
\varepsilon_{\chi \mathrm{PT}}=-p+\mu n_{q}=4 N_{f} f_{\pi}^{2}\left(\mu^{2}-3 \frac{\mu_{o}^{4}}{\mu^{2}}+2 \mu_{o}^{2}\right) ; \\
\left(T_{\mu \mu}\right)_{\chi \mathrm{PT}}=\varepsilon-3 p=8 N_{f} f_{\pi}^{2}\left(-\mu^{2}-3 \frac{\mu_{o}^{4}}{\mu^{2}}+4 \mu_{o}^{2}\right) .
\end{gathered}
$$

Note that the trace of the stress-energy tensor $\left(T_{\mu \mu}\right)_{\chi \mathrm{PT}}<$ 0 for $\mu>\sqrt{3 \mu_{o}}$.

These model results should be contrasted with those of another paradigm for cold dense matter, namely, a degenerate system of weakly interacting (thus presumably deconfined) quarks populating a Fermi sphere up to some maximum momentum $k_{F} \approx E_{F}=\mu$ :

$$
n_{\mathrm{SB}}=\frac{N_{f} N_{c}}{3 \pi^{2}} \mu^{3} ; \quad \varepsilon_{\mathrm{SB}}=3 p_{\mathrm{SB}}=\frac{N_{f} N_{c}}{4 \pi^{2}} \mu^{4} .
$$

In this picture superfluidity arises from condensation of diquark Cooper pairs from within a layer of thickness $\Delta$ centered on the Fermi surface; hence

$$
\langle q q\rangle \propto \Delta \mu^{2} .
$$

Since $p_{\mathrm{SB}}$ eventually exceeds $p_{\chi \mathrm{PT}}$ as $\mu$ increases, the degenerate system must be the more thermodynamically stable at high density. In fact, since both $n_{q}$ and $\varepsilon$ are discontinuous at the point where $p_{\mathrm{SB}}=p_{\chi \mathrm{PT}}$, this naive treatment predicts the resulting deconfining transition is first order [6].

These considerations have motivated us to pursue lattice simulations of $\mathrm{QC}_{2} \mathrm{D}$ beyond the BEC regime, using $N_{f}=$ 2 flavors of Wilson fermion. The quark action is

$$
S=\sum_{i=1,2} \bar{\psi}_{i} M \psi_{i}+\kappa j\left[\psi_{2}^{\operatorname{tr}}\left(C \gamma_{5}\right) \tau_{2} \psi_{1}-\text { H.c. }\right]
$$

with

$$
\begin{aligned}
M_{x y}= & \delta_{x y}-\kappa \sum_{\nu}\left[\left(1-\gamma_{\nu}\right) e^{\mu \delta_{\nu 0}} U_{\nu}(x) \delta_{y, x+\hat{\nu}}\right. \\
& \left.+\left(1+\gamma_{\nu}\right) e^{-\mu \delta_{\nu 0}} U_{\nu}^{\dagger}(y) \delta_{y, x-\hat{\nu}}\right] .
\end{aligned}
$$

A conventional Wilson action was used for the glue fields. Further details can be found in [6].

Since Wilson fermions do not have a manifest chiral symmetry, we have little to say at this stage about this aspect of the physics, which at high quark density should be of secondary importance for phenomena near the Fermi surface; they do, however, carry a conserved baryon charge due to the $\mathrm{U}(1)_{B}$ symmetry $\psi \mapsto e^{i \alpha} \psi, \bar{\psi} \mapsto \bar{\psi} e^{-i \alpha}$. Our initial runs on a $8^{3} \times 16$ lattice with $\beta=1.7, \kappa=0.178$ corresponding to lattice spacing $a=0.230(5) \mathrm{fm}, m_{\pi} a=$ $0.79(1)$, and $m_{\pi} / m_{\rho}=0.779(4)$ have been described in [6]. In this Letter we present data from runs on an approximately matched $12^{3} \times 24$ lattice with $\beta=1.9, \kappa=0.168$ corresponding to $a=0.186(8) \mathrm{fm}, \quad m_{\pi} a=$ $0.68(1)$, and $m_{\pi} / m_{\rho}=0.80(1)$. The physical scale is set by equating the observed string tension at $\mu=0$ to $(440 \mathrm{MeV})^{2}$. Note that the physical temperature $T$ is approximately 54(1) $\mathrm{MeV}$ for the smaller lattice, and 44(2) MeV for the larger. We used a standard HMC algorithm-the only novelty is the inclusion of a diquark source term [proportional to $j$ in Eq. (8)]; this mitigates the impact of IR fluctuations in the superfluid regime and also enables the algorithm to change the sign of $\operatorname{det} M$ for a single flavor, thus maintaining ergodicity. All results presented here were obtained with $j a=0.04$; ultimately the physical limit $j \rightarrow 0$ must be taken.

Figure 1 shows results for quark density and pressure as functions of $\mu$, plotted as ratios of the same quantities evaluated for free massless quarks on the same lattice [6]. In the $j \rightarrow 0$ limit the onset is expected at $\mu_{o} a=0.34$ corresponding to $\mu_{o} \simeq 360 \mathrm{MeV}$; the observation of $n_{q}$, $p>0$ for $\mu<\mu_{o}$ is an artifact of working with $j \neq 0$.

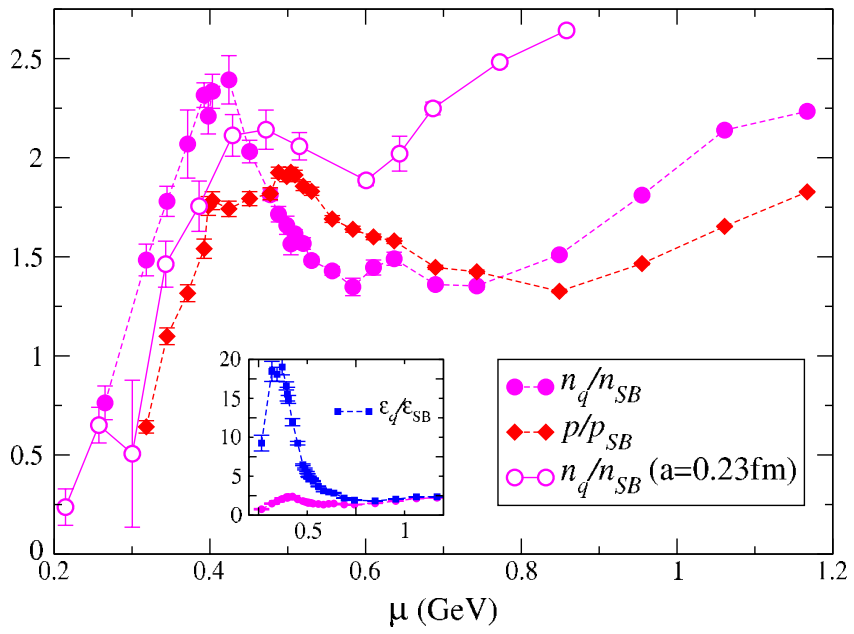

FIG. 1 (color online). $\quad n_{q} / n_{\mathrm{SB}}$ and $p / p_{\mathrm{SB}}$ vs $\mu$ for $\mathrm{QC}_{2} \mathrm{D}$. Inset shows $\varepsilon_{q} / \varepsilon_{\mathrm{SB}}$ for comparison. 
Beyond onset the ratio $n_{q} / n_{\mathrm{SB}}$ rises to a peak at $\mu \approx$ $400 \mathrm{MeV}$, and then falls to a plateau beginning at $\mu_{Q} \approx$ $530 \mathrm{MeV}$, which continues until $\mu_{D} \approx 850 \mathrm{MeV}$ where it starts to rise again. If following the arguments presented above we associate the plateau with the setting in of degenerate matter, then we identify a BEC/BCS crossover at $\mu_{Q} \approx 530 \mathrm{MeV}$, corresponding to a quark density $n_{q} \approx$ 4-5 $\mathrm{fm}^{-3}$, i.e. roughly 10 times nuclear density. In order to specify these numbers with greater precision, as well as the statistical errors manifest in the error bars of Fig. 1, we need to establish control over lattice artifacts by taking the continuum limit $a \rightarrow 0$. So far we have data from only two lattice spacings; results for $n_{q} / n_{\mathrm{SB}}$ taken with $a=0.23 \mathrm{fm}$ plotted in Fig. 1 show that there is reasonable scaling for $\mu \lesssim 400 \mathrm{MeV}$-indeed, the small difference in the physical $j$ between the two ensembles has maximal impact for $\mu \approx \mu_{o}[5,6]$. The situation at larger $\mu$ will be discussed further below.

In contrast to $\chi \mathrm{PT}$, the quark contribution to the energy density $\varepsilon_{q}$ exceeds the free field value by almost a factor of 20 for $\mu \gtrsim \mu_{o}$, as shown in the inset of Fig. 1; it should be remarked here that unlike $n_{q}$ and $p, \varepsilon$ is subject to a multiplicative quantum correction known as a Karsch coefficient [7] which is still to be calculated for this system, though its renormalized value is unlikely to differ by more than $50 \%$. In any case, since the Karsch coefficient is $\mu$ independent, the shape of the curve will remain the same. Because of this unexpected behavior at small $\mu$, the energy per quark $\varepsilon_{q} / n_{q}$ exhibits a shallow but robust minimum for $\mu>\mu_{Q}$, a feature completely absent in the model governed by Eqs. (2)-(6).

Figure 2 plots the conformal anomaly $T_{\mu \mu}=\left(T_{\mu \mu}\right)_{g}+$ $\left(T_{\mu \mu}\right)_{q}$, with

$$
\left(T_{\mu \mu}\right)_{g}=-\left.a \frac{\partial \beta}{\partial a}\right|_{\mathrm{LCP}} \times \frac{3 \beta}{N_{c}} \operatorname{Tr}\left\langle\square_{t}+\square_{s}\right\rangle ;
$$

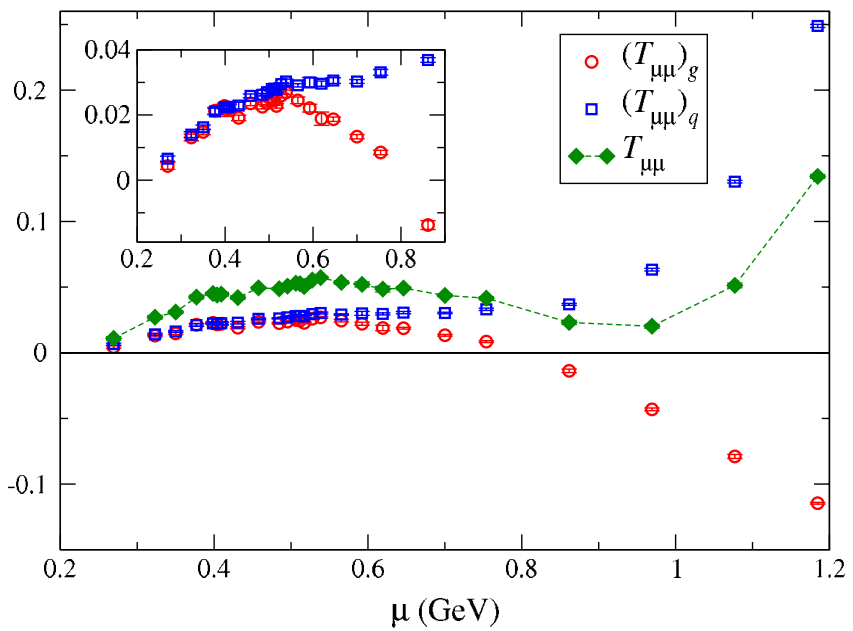

FIG. 2 (color online). Conformal anomaly $T_{\mu \mu} a^{4}$ vs $\mu$, plotted together with separate quark and gluon contributions.

$$
\left(T_{\mu \mu}\right)_{q}=\left.a \frac{\partial \kappa}{\partial a}\right|_{\mathrm{LCP}} \times \kappa^{-1}\left(4 N_{f} N_{c}-\langle\bar{\psi} \psi\rangle\right),
$$

where LCP denotes the beta function is determined along a line of constant physics, and once again a vacuum contribution must be subtracted. The required beta functions are estimated from the parameters of the two matched lattices to be $-0.85(17)(g)$ and $0.042(9)(q)$ (these errors are not included in Fig. 2).

Figure 2 shows that for $\mu \lesssim \mu_{Q}$ the contribution of both quarks and gluons to $T_{\mu \mu}$ is initially positive and increasing with $\mu$; here both quarks and gluons are contained within tightly bound nonrelativistic bosons, implying that $\varepsilon>3 p$. For $\mu \gtrsim \mu_{Q}$, however, their behavior diverges sharply (see inset), which could possibly be explained by quark and gluon degrees of freedom now being governed by differing quantum statistics. In fact, the gluon data are very well approximated over the whole $\mu$ range by a parabola, and $\left(T_{\mu \mu}\right)_{g}$ accordingly becomes negative for $\mu \gtrsim \mu_{D}$. This change in sign has also been predicted using $\chi \mathrm{PT}$ and the property of asymptotic freedom [8] [another way of understanding the necessity for $\left(T_{\mu \mu}\right)_{g}$ to change sign is that the plaquette must revert to its quenched value in the limit $\mu \rightarrow \infty$ where quantum corrections due to quarks are Pauli blocked [6] ]. At the same point there is a very sharp change in the behavior of $\left(T_{\mu \mu}\right)_{q}$, which had been approximately constant for $\mu_{Q} \lesssim \mu \lesssim \mu_{D}$. Figure 2 shows that at large $\mu$ the quark contribution dominates, so that $\lim _{\mu \rightarrow \infty} T_{\mu \mu}>0$. This behavior is not predicted by $\chi$ PT [e.g. Eq. (5) and Ref. [8] ], although the positivity of $T_{\mu \mu}$ in this limit is consistent with three-loop perturbation theory [9].

Next consider the gluonic energy density given by

$$
\varepsilon_{g}=\frac{3 \beta}{N_{c}} \operatorname{Tr}\left\langle\square_{t}-\square_{s}\right\rangle
$$

(once again, a $\mu$-independent Karsch coefficient is still to be determined). Figure 3 plots the dimensionless combination $\varepsilon_{g} / \mu^{4}$ against $\mu$; of course, this quantity is not predicted in either $\chi \mathrm{PT}$ or the free quark gas. While we therefore have no quantitative theory of the gluonic contribution to $\mathrm{QC}_{2} \mathrm{D}$ thermodynamics at $\mu \neq 0$, we would expect its relative importance to increase across a deconfining transition. In fact, the ratio is remarkably constant over a wide range of $\mu$, consistent with dimensional analysis; in particular, there is no sign of singular behavior at $\mu=\mu_{Q}$, although there is a systematic rise for $\mu \gtrsim \mu_{D}$.

Figure 4 plots quantities giving information on the nature and symmetries of the ground state. In the limit $j \rightarrow 0$, the diquark condensate $\langle q q\rangle$ is an order parameter for the spontaneous breaking of $\mathrm{U}(1)_{B}$ leading to baryon number superfluidity. Although the data of Fig. 4 are taken with $j \neq 0$, implying some care must be taken with the extrapolation $j \rightarrow 0$ at small $\mu$ [6], we are confident that this symmetry is broken for all $\mu>\mu_{o}$. The approximate flat- 


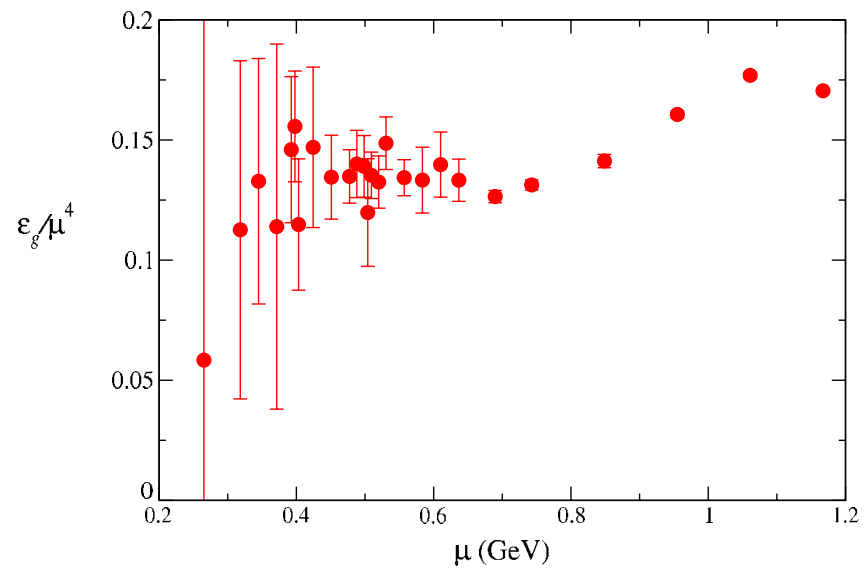

FIG. 3 (color online). Gluon energy density $\varepsilon_{g} / \mu^{4}$ vs $\mu$.

ness of the curve for $\mu_{Q} \lesssim \mu \lesssim \mu_{D}$ is then evidence for a scaling $\langle q q\rangle \propto \mu^{2}$ similar to Eq. (7). We take this as an indication that in this region the system consists of degenerate quark matter with a Fermi surface disrupted by a BCS instability.

The Polyakov line is an order parameter for deconfinement in the limit of infinitely massive quarks-away from this limit it continues to yield information on the free energy of an isolated color source. Figure 4 shows that $\mathrm{QC}_{2} \mathrm{D}$ remains confined for $\mu<\mu_{D}$, but that there appears to be a transition to a deconfined state for chemical potentials in excess of this value. In physical units $\mu_{D} \approx$ $850 \mathrm{MeV}$, corresponding to quark density $n_{q} \approx$ 16-32 $\mathrm{fm}^{-3}$, some 35-70 times nuclear density.

To summarize, the simulations suggest that $\mathrm{QC}_{2} \mathrm{D}$ has three distinct transitions (or at least crossovers). The first, at $\mu=\mu_{o}$, is a firmly established second order phase transition (in the limit $j \rightarrow 0$ ) from vacuum to a BEC superfluid and is described accurately for the most part

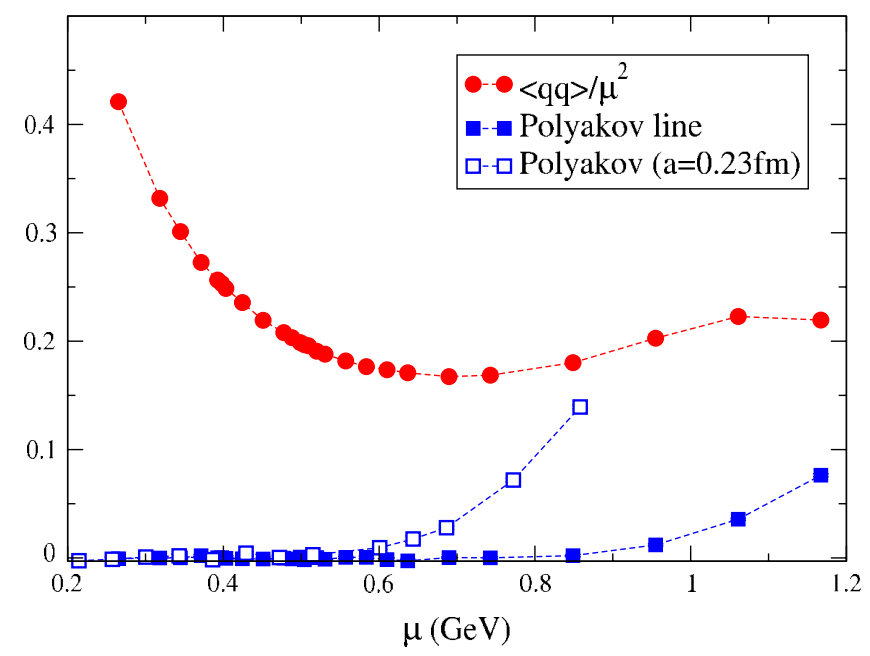

FIG. 4 (color online). Superfluid order parameter $\langle q q\rangle / \mu^{2}$ and Polyakov line vs $\mu$. by $\chi \mathrm{PT}$ (the quark energy density $\varepsilon_{q}$ looks to be an important exception). Since the pion is not especially light with our choice of lattice parameters, implying only a moderate separation of Goldstone and hadronic mass scales, the $\mu$ window within which the BEC is favored is not particularly wide.

The second transition at $\mu=\mu_{Q}$ looks like a BEC/BCS crossover to form a ground state where the scalings of the observables $n_{q}(\mu), p(\mu), \varepsilon_{q}(\mu)$, and $\langle q q(\mu)\rangle$ all suggest it is formed of degenerate quark matter with a well-defined Fermi sphere, albeit one whose surface is disrupted by a BCS condensate. We note that effective treatments based on both $\chi \mathrm{PT}$ and Nambu-Jona-Lasinio (NJL) models predict $n_{q} / n_{\mathrm{SB}}$ to be monotonic decreasing in this regime and are unable to fit the lattice data [10]. The distinct nature of this region is also supported by the diverging behaviors of $\left(T_{\mu \mu}\right)_{g}$ and $\left(T_{\mu \mu}\right)_{q}$ for $\mu \gtrsim \mu_{Q}$ seen in Fig. 2, although the reason for the peculiar behavior of $\left(T_{\mu \mu}\right)_{q}$ is not well understood at present. The transition at $\mu \approx \mu_{Q}$ is most likely a smooth crossover, but the exact nature of this putative transition requires further study, in particular, a careful extrapolation to the limit of zero diquark source.

The third transition at $\mu=\mu_{D}$ is signaled by a change in the scaling of the thermodynamic observables, notably $\varepsilon_{g}(\mu)$ and $\left(T_{\mu \mu}\right)_{q}$, a change in the sign of $\left(T_{\mu \mu}\right)_{g}$, and a nonzero Polyakov loop. For $\mu>\mu_{D}$ the system consists of deconfined quark matter.

An immediate concern is the validity of the deconfining transition in the continuum limit. Figure 4 shows that with $a=0.23 \mathrm{fm}, \mu_{D} \approx 600 \mathrm{MeV}$, and is practically indistinguishable from $\mu_{Q}$; for this reason only a deconfined quark matter phase was identified in Ref. [6] (cf. Figure 1). In both cases, however, the quark density in lattice units $n_{q} a^{3}=0.17$ (coarse) or 0.20 (fine), well short of the value $2 N_{c} N_{f}$ signifying that lattice saturation artifacts have set in-indeed for $a=0.186 \mathrm{fm}$ trends in all observables look smooth out to $\mu a=1.0$ corresponding to $\mu=1.06 \mathrm{GeV}$. It is therefore plausible that the observed difference in $\mu_{D}$ is physical, and due to the differing temperatures of the two lattices used.

Between $\mu_{Q}$ and $\mu_{D}$ the system resembles the quarkyonic matter recently postulated on the basis of large- $N_{c}$ arguments [2], namely, a state of degenerate matter which is also confined, so that excitations above the ground state remain color singlet. Because we have used Wilson fermions (with no manifest chiral symmetry), we are unfortunately unable at this point to test whether chiral symmetry is restored, another important aspect of the quarkyonic hypothesis; we note, however, that even in a conventional scenario $\chi$ PT predicts $\langle\bar{\psi} \psi\rangle \propto \mu^{-2}$ for $\mu \geq \mu_{o}$ [5], which is likely to be difficult to distinguish from true chiral symmetry restoration in this region, particularly if the transition is a crossover.

The apparent sensitivity of the value of $\mu_{D}$ to small changes in temperature is consistent with the very weak 


\section{QUARKYONIC PHASE IN DENSE TWO COLOR MATTER}

curvature of the phase boundary between confined and deconfined phases postulated in that work, and observed in a recent study of $\mathrm{QC}_{2} \mathrm{D}$ matter using the PolyakovNambu-Jona-Lasinio (PNJL) model [11].

An interesting issue is whether $\mathrm{QC}_{2} \mathrm{D}$ is special in that the $N_{c}$-quark bound states required by color confinement are also favored by the more general renormalization group argument that 2-body interactions are the only relevant ones close to a Fermi surface [12]. $\mathrm{QC}_{2} \mathrm{D}$ is also exceptional, of course, because since $\langle q q\rangle$ is gauge singlet there
PHYSICAL REVIEW D 81, 091502(R) (2010)

is no CSC phase. Whatever the outcome, to our mind the study of deconfinement in this hitherto-unexplored physical regime promises to be fascinating.

This project was enabled with the assistance of IBM Deep Computing. S. K. was supported by the National Research Foundation of Korea funded by the Korea government (MEST) Grant No. 2009-0074027. We benefited greatly from discussions with Ernst-Michael Ilgenfritz.
[1] M. G. Alford, A. Schmitt, K. Rajagopal, and T. Schafer, Rev. Mod. Phys. 80, 1455 (2008).

[2] L. McLerran and R. D. Pisarski, Nucl. Phys. A796, 83 (2007).

[3] T. D. Cohen, Phys. Rev. Lett. 91, 222001 (2003).

[4] S. J. Hands, I. Montvay, S. E. Morrison, M. Oevers, L. Scorzato, and J. I. Skullerud, Eur. Phys. J. C 17, 285 (2000).

[5] J. B. Kogut, M. A. Stephanov, D. Toublan, J. J. M. Verbaarschot, and A. Zhitnitsky, Nucl. Phys. B582, 477 (2000).

[6] S. J. Hands, S. Kim, and J. I. Skullerud, Eur. Phys. J. C 48, 193 (2006).
[7] There is also an additive correction that has been subtracted by requiring $\varepsilon(\mu=0)=0$.

[8] M. A. Metlitski and A. R. Zhitnitsky, Nucl. Phys. B731, 309 (2005).

[9] A. Kurkela, P. Romatschke, and A. Vuorinen, arXiv:0912.1856 [Phys. Rev. D (to be published).

[10] J. O. Andersen and T. Brauner, arXiv:1001.5168 [Phys. Rev. D (to be published).

[11] T. Brauner, K. Fukushima, and Y. Hidaka, Phys. Rev. D 80, 074035 (2009).

[12] R. Shankar, Rev. Mod. Phys. 66, 129 (1994); J. Polchinski, arXiv:hep-th/9210046. 